$\left.r_{s}=0.29\right)$, urge to cough VAS $\left(r_{s}=0.24\right)$ and LCQ $\left(r_{s}=0.32\right)$, all $\mathrm{p}>0.10$.

Conclusion Voluntary suppression of capsaicin-evoked cough is significantly diminished in chronic refractory cough. Our findings suggest future research should focus on cough inhibitory as well as activation pathways. CS5 has potential to be used as a diagnostic test and to evaluate anti-tussive therapy; this should be investigated further.

\section{S33 THE UTILITY OF FENO IN THE DIFFERENTIAL DIAGNOSIS OF CHRONIC COUGH: THE RESPONSE TO ANTI- INFLAMMATORY THERAPY WITH PREDNISOLONE AND MONTELUKAST}

M Haji Sadeghi, C Wright, S Hart, M Crooks, A Morice. Hull York Medical School, Hull, UK

\subsection{6/thoraxjnl-2017-210983.39}

Objectives In this study we explored the effectiveness of treatment with montelukast $10 \mathrm{mg}$ as compared with prednisolone in chronic cough patients with an associated elevated FeNO (The fraction of exhaled nitric oxide in breath) - a marker of eosinophilic inflammation.

Methods 50 non-asthmatic patients with chronic cough were recruited sequentially from a specialist cough clinic. 30 patients with high FeNO $(\geq 30 \mathrm{ppb})$ were randomised to either two weeks prednisolone $20 \mathrm{mg}$ or two weeks montelukast $10 \mathrm{mg}$ followed by montelukast $10 \mathrm{mg}$ for the subsequent two weeks in both arms. A control group of 20 patients with low FeNO ( $\leq 20 \mathrm{ppb})$ were enrolled who received four weeks montelukast. 24 hours cough counting at baseline after 2 and 4 weeks treatment was the primary endpoint. Subjective measures of cough, the Leicester Cough Questionnaire (LCQ), and Hull Airways Reflux Questionnaire (HARQ) were also administered.

Results At baseline the average FeNO value in both high FeNO treatment groups was similar (around $60 \pm 30 \mathrm{ppb}$ ). At the end of the study there was a significant fall in FeNO of approximately $30 \%$ in both high FeNO treatment groups ( $\mathrm{p}$ $<0.005)$. In the low FeNO group there was no significant change during the study $(12 \pm 5 \mathrm{ppb})$. Therapy reduced the number of coughs in 24 hours by approximately 50\% in both low and high FeNO groups $(\mathrm{p}<0.005)$. HARQ and LCQ scores also improved significantly $(\mathrm{p}<0.005)$ in all treatment groups.

Conclusions The hypothesis that FeNO could be used as a marker of eosinophilic inflammation in chronic cough was supported by our observation at baseline in the high FeNO group of eosinophilia in both blood and sputum. However, baseline FeNO did not predict overall treatment response. Perhaps the most surprising aspect of our study is the dramatic response in the low FeNO group to montelukast. The fact
FENO

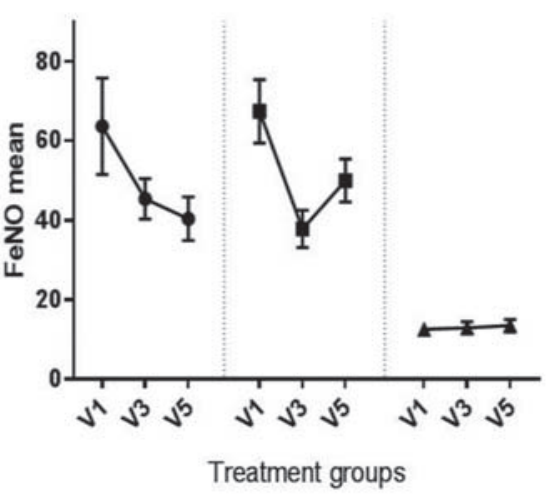

HARQ

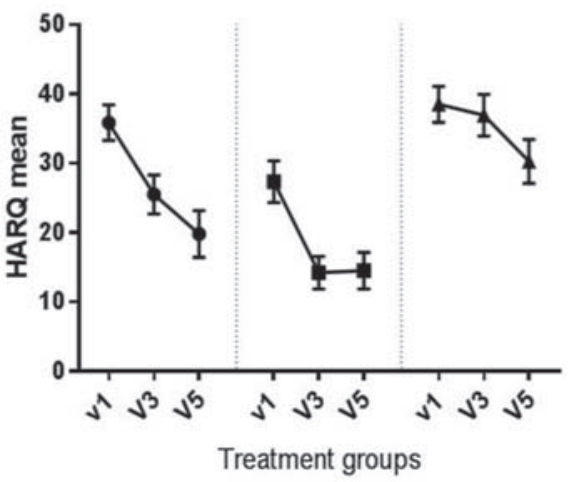

$24 \mathrm{hr}$ C.C

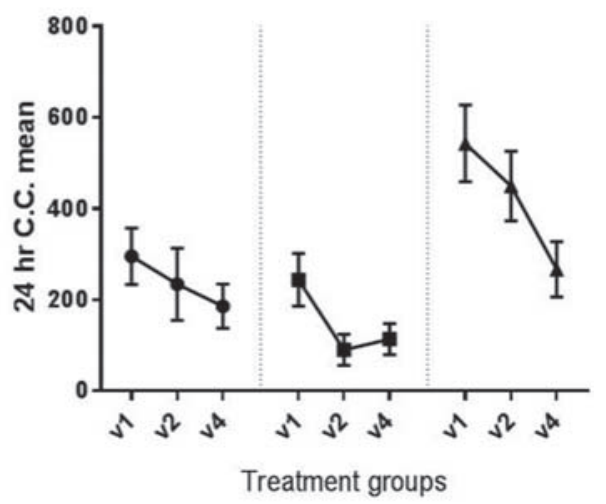

LCQ

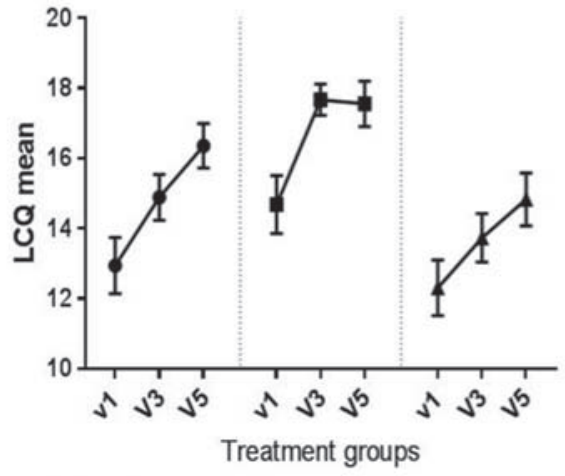

- High FeNO group arm1 (montelukast)

- High FeNO group arm2 (prednisolone + montelukast)

$\Delta$ Low FeNO group (montelukast)

Abstarct S33 Figure 1 Measurements of FeNO, 24hr cough count, HARQ and LCQ in three treatment groups in three visits. Horizontal bars represent mean and SEM value. 
that montelukast appears to be equally effective in the low FeNO group suggest the either the current markers of eosinophilic lung disease are insufficiently sensitive to pick up low levels of leukotriene activation in the low FeNO group, or that montelukast has its antitussive activity by an alternative mechanism.

\section{S34 THE EFFECTS OF A NOVEL FORMULATION OF INHALED CROMOLYN SODIUM (PA101) IN IDIOPATHIC PULMONARY FIBROSIS AND CHRONIC COUGH: A RANDOMISED, CONTROLLED, PHASE 2 TRIAL}

${ }^{1}$ SS Brring, ${ }^{2} \mathrm{MS}$ Wijsenbeek, ${ }^{3} \mathrm{~S}$ Agrawal, ${ }^{4} \mathrm{JWK}$ van den Berg, ${ }^{5} \mathrm{H}$ Stone, ${ }^{6} \mathrm{TM}$ Maher, ${ }^{7} \mathrm{~A}$ Tutuncu, ${ }^{8} \mathrm{AH}$ Morice. 'Division of Asthma, Allergy and Lung Biology, King's College London, London, UK; ' ${ }^{2}$ Dept. Respiratory Medicine, Erasmus University Medical Centre, Rotterdam, The Netherlands; ${ }^{3}$ Dept. Respiratory Medicine, Glenfield Hospital, Leicester, UK; ${ }^{4}$ Dept. Pulmonology, Isala Hospital, Zwolle, Netherlands; ${ }^{5}$ Dept. Respiratory Medicine, Royal Stoke University Hospital, Stoke-on-Trent, UK; ${ }^{6}$ Royal Brompton Hospital and Fibrosis Research Group, National Heart and Lung Institute, Imperial College, London, UK; ${ }^{7}$ Patara Pharma, San Diego, California, US; ${ }^{8}$ Hull York Medical School, Castle Hill Hospital, Hull, UK

\subsection{6/thoraxjnl-2017-210983.40}

Introduction and Objectives Cough can be a debilitating symptom of idiopathic pulmonary fibrosis (IPF). The efficacy and safety of inhaled PA101, a novel formulation of cromolyn sodium was investigated in IPF patients with chronic cough in a multi-centre, randomised, double-blind, placebo-controlled, 2-cohort, 2-period, cross-over trial. To explore the mechanism of anti-tussive activity of PA101, a parallel study of similar design was conducted in patients with chronic idiopathic cough (CIC).

Methods Twenty-four participants with IPF and chronic cough were randomised to receive PA101 $(40 \mathrm{mg})$ or matching placebo three times a day for 2 weeks, followed by 2 weeks wash out, and then crossed over. The primary outcome measure was objective daytime cough frequency (from 24 hour acoustic recording, Leicester Cough Monitor) and secondary outcomes included subjective cough-specific quality of life (LCQ) assessed at Day 14. The primary efficacy analysis was based on a mixed effects model. In the CIC cohort, twentyeight participants were randomised in a study with similar design and endpoints.

Results In IPF patients, PA101 reduced daytime cough frequency by $31.1 \%$ at day 14 compared to placebo. Daytime cough frequency decreased from a mean(SD) of 55(55) to 39 (29) coughs/hour at day 14 with PA101 versus 51(37) to 52 (40) coughs/hour following placebo (figure 1). There was a significant correlation coefficient between change in cough severity (VAS score) and change in daytime cough frequency with PA101 at Day 14, $r=0.415 \quad(p=0.048)$. There was a trend towards improvement in LCQ score with PA101 compared to placebo; mean treatment difference 1.1, $\mathrm{p}=0.09$. PA101 was associated with an improvement in chest symptom and psychological domains of the KBILD-QOL $(p<0.05)$. In contrast, no treatment benefit for PA101 was observed in the CIC cohort; mean reduction of daytime cough frequency at Day 14 for PA101 adjusted for placebo was 6.2\%. PA101 was well tolerated in both cohorts and the incidence of adverse events was comparable between PA101 and placebo treatments.

Conclusions This study suggests that the mechanism of cough in IPF may be disease specific. Inhaled PA101 may be a treatment option for chronic cough in patients with IPF and warrants further investigation.

\section{S35 RANDOMISED CONTROL TRIAL QUANTIFYING THE EFFICACY OF LOW DOSE MORPHINE IN A RESPONDER GROUP OF PATIENTS WITH REFRACTORY CHRONIC COUGH}

'B Al-Sheklly, ${ }^{2}$ J Mitchell, 'B Issa, ${ }^{1} \mathrm{H}$ Badri, ${ }^{2} \mathrm{~S}$ Satia, ${ }^{2} \mathrm{~T}$ Collier, ${ }^{1} \mathrm{~S}$ Sen, ${ }^{1} \mathrm{~J}$ Webber Ford, ${ }^{1} \mathrm{D}$ Corfield, ${ }^{1} \mathrm{JA}$ Smith. ${ }^{1}$ University of Manchester, Manchester, UK; ${ }^{2}$ University Hospital South Manchester, Manchester, UK

\subsection{6/thoraxjnl-2017-210983.41}

Introduction and objectives The pathophysiology of chronic cough remains poorly understood and treatment options are limited. Morphine sulphate can improve quality of life in refractory chronic cough, ${ }^{1}$ but its mechanism of action and effect on objective cough frequency remain unknown. Here we report morphine's influence on cough frequency and patient reported outcomes in a group of refractory chronic cough patients who responded favourably to morphine in our tertiary clinic.

Methods We recruited 22 patients (mean age 61.7 years, 18 female, mean cough duration 14 years) taking low dose morphine for refractory chronic cough into a double blinded, placebo controlled, crossover study. Volunteers withdrew their morphine therapy and were randomised to receive morphine (5-10 mg BD slow release)/matched placebo during two treatment period (5-7 days duration) separated by a 5-7 day washout. At baseline and at the end of each treatment period we assessed 24 hour cough recordings using a semi-automated cough counter (vitaloJAK), cough severity (visual analogue

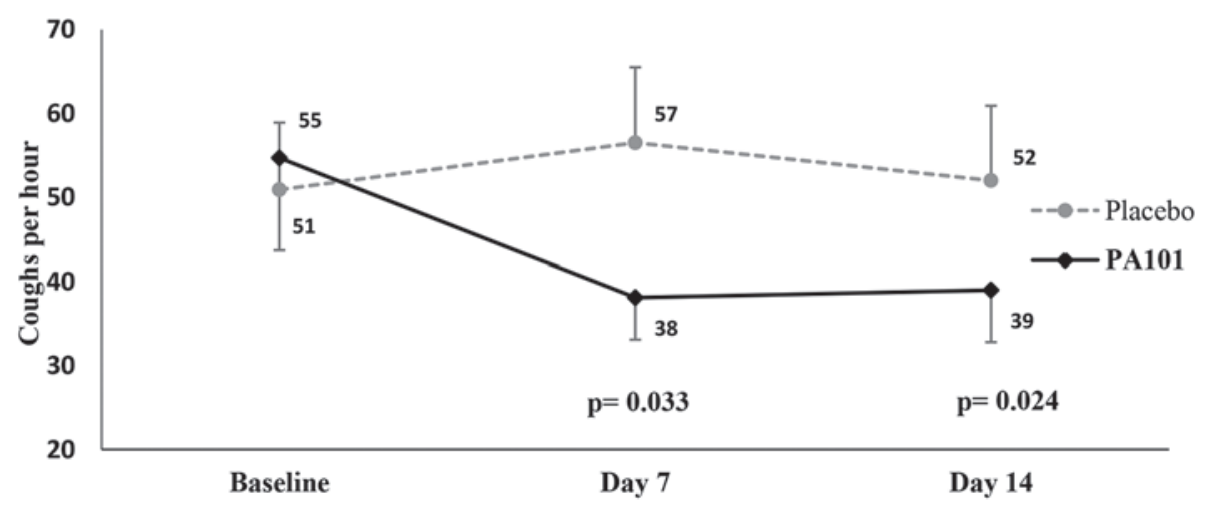

Abstract S34 Figure 1 Changes in daytime cough frequency in IPF cohort. 